Check for updates

Cite this: J. Anal. At. Spectrom., 2017 32,2455

Received 4th September 2017

Accepted 1st November 2017

DOI: $10.1039 / c 7 j a 00306 d$

rsc.li/jaas

\section{Dimensional characterization of gold nanorods by combining millisecond and microsecond temporal resolution single particle ICP-MS measurements $\uparrow$}

\author{
Ildikó Kálomista, ${ }^{a}$ Albert Kéri, (iD a Ditta Ungor, ${ }^{b}$ Edit Csapó, ${ }^{b c}$ Imre Dékány, \\ Thomas Prohaska (D) and Gábor Galbács (D) *a
}

\begin{abstract}
A systematic investigation of single particle inductively coupled plasma mass spectrometry (splCP-MS) signal profiles recorded with normal (ms-range) and high ( $\mu$ s-range) temporal resolution for spherical and rod-shaped gold nanoparticles was performed. The experiments with nanorods were carried out on hemispherically capped cylindrical particles synthesized in the aspect ratio range from 1.5 to 4.5. A comparison of NP signals and time profiles for spherical and rod-shaped NPs revealed that (i) the volume of the particles can be assessed by conventional spICP-MS measurements using a joint, linear calibration plot; and (ii) the shape of signal time profiles and transit times for equal-volume spherical and rodshaped NPs are different, based on which the aspect ratio can be calculated. By using the statistical evaluation of the high resolution signal time profiles, an analytical method was developed for the discrimination and dimensional analysis of nanorod-shaped NPs. The method is based on observing the shortest and longest transit times for nanoparticles. In the case of spherical NPs, these transit times are very similar, whereas in the case of nanorods, the shortest and longest transit times correlate with the characteristic width and length of the particles, respectively. This method is the first nanorod dimensional characterization method in the literature, which is based solely on ICP-MS measurements. It was shown that the accuracy of the method is very good - the characteristic length and width of the particles could be determined with less than $6 \%$ error.
\end{abstract}

\section{Introduction}

Nanoscience is a rapidly upcoming field in science and technology. Nanomaterials (nanoparticles, nanocomposites, nanostructures, etc.) are nowadays widely used in a broad range of application fields from medicine to energetics and sensorics to catalysis. ${ }^{1-4}$ Initially, isotropic or (quasi-)spherical nanoparticles (NPs) were used in applications due to the ease of their synthesis. Later, as the synthesis of anisotropic NPs became more efficient and reproducible, ${ }^{5,6}$ the exploration of the unique mechanical and optical properties of these structures as well as their utility also started. ${ }^{7}$

The first anisotropic nanoparticles to receive attention were gold and silver nanorods, but especially gold, due to its

\footnotetext{
${ }^{a}$ Department of Inorganic and Analytical Chemistry, University of Szeged, Dóm square 7, 6720 Szeged, Hungary.E-mail: galbx@chem.u-szeged.hu

${ }^{b}$ Department of Physical Chemistry and Materials Science, University of Szeged, Aradi V. square 1, 6720 Szeged, Hungary

'MTA-SZTE Biomimetic Systems Research Group, Department of Medical Chemistry, University of Szeged, Dóm square 8, 6720 Szeged, Hungary

${ }^{d}$ Division of Analytical Chemistry, University of Natural Resources and Life Sciences, Gregor-Mendel-Straße 33, 1180 Vienna, Austria

$\dagger$ Electronic supplementary information (ESI) available. See DOI: 10.1039/c7ja00306d
}

biocompatibility. ${ }^{8,9}$ It was found that even the simplest anisotropic geometry, the rod shape, offers benefits over spheroidal NPs in terms of their tunable surface plasmon resonance (SPR), namely, they display two SPR absorption bands (known as transverse and longitudinal plasmon bands), and while the transverse SPR band is always located in the visible part of the electromagnetic spectrum (at around 500-600 $\mathrm{nm}$ ), the peak wavelength of the longitudinal band strongly depends on the aspect ratio. ${ }^{10,11}$ Thus nanorods can have widely tunable light absorption anywhere in the visible or NIR range, which is a valuable property e.g. in biomedical or sensor applications (allowing for a boost of selectivity or sensitivity). ${ }^{6,12}$

Nowadays, most gold nanorods used are crystalline or polycrystalline and are synthesized in an aqueous medium, using cetyltrimethylammonium bromide (CTAB) mediated procedures or by reduction of gold inside some solid template such as anodized aluminum oxide. ${ }^{6,12}$ The structural characterization (determination of the shape, size and aspect ratio) of the nanorods is typically done by using transmission electron microscopy (TEM) and UV-vis spectroscopy. ${ }^{10}$

In single particle (or particle-mode) inductively coupled plasma mass spectrometry (spICP-MS), the ICP-MS is used in the time-resolved mode for the measurement of nanodispersions intentionally so dilute that each particle is detected 
separately as a narrow signal peak with a height that is proportional to the mass (number of atoms) of the analyte in the NP. The time resolution is normally $5-10 \mathrm{~ms}$, which is available on most modern ICP-MS spectrometers. By statistical evaluation of the time-resolved signal, information can be obtained about not only the elemental (isotopic) composition of the NPs, but also their size distribution, as well as the particle concentration. For monometallic NPs, the typical size detection limits range from 10 to $30 \mathrm{~nm}$ depending on the sensitivity of the instrument and the abundance of the measured isotope. ${ }^{\mathbf{1 3 - 1 6}}$ Recently, quadrupole ICP-MS instruments with fast data acquisition rates allowing for 10-20 microsecond dwell times ("high time resolution") became available commercially. Since the duration of the NP signal peak in an ICP-MS is in the hundreds of microseconds range, the peak can be adequately resolved in high resolution spICP-MS (HR-spICP-MS). ${ }^{17-19}$ In addition, the dynamic range of spICP-MS measurements can also be extended. ${ }^{19}$ Thus, high time resolution can potentially extend the capabilities of the spICP-MS technique.

The fundamental question behind the present study was whether the combination of normal and high time resolution spICP-MS measurements could provide information about the shape and dimensions of NPs or not. Our working hypothesis was that while the mean size (volume) of the particles can be estimated from normal time resolution spICP-MS measurements, the differences between the HR signal time profiles for spherical and rod-shaped NPs could be related to the aspect ratio. The assessment was done by examining the signal time profiles for spherical gold NPs and gold nanorods of various sizes and aspect ratios. Gold NP standards and gold nanorods specifically synthesized by wet-chemical methods in our laboratory for the purpose of the present study were used in this investigation.

\section{Experimental}

\subsection{ICP-MS instrumentation}

Two ICP-MS instruments were used in the time resolved analysis (TRA) measurements. Normal resolution (millisecond integration time) spICP-MS measurements were performed mostly on an Agilent $7700 \times$ type instrument (University of Szeged, Szeged, Hungary), while the high resolution (microsecond integration time) HR-spICP-MS measurements were all carried out on a PerkinElmer NexION 350 type instrument (University of Natural Resources and Life Sciences, Vienna, Austria). On the NexION 350, the Nano software module (Syngistix Nano Application Module) was used for data collection. The instrumental settings and conditions are summarized in Table 1.

\subsection{Gold nanorods and nanospheres}

All reagents used in the synthesis and nanodispersion preparation were of analytical purity, except for deionized water, which was of trace analytical purity (MilliPore Elix 5 with Synergy, USA). In every case, the nanodispersions were prepared with $10^{5} \mathrm{~mL}^{-1}$ particle concentration and were always sonicated
Table 1 ICP-MS instrumental settings and conditions

\begin{tabular}{lll}
\hline Parameter/device & NexION 350 & Agilent $7700 \times$ \\
\hline RF power & $1400 \mathrm{~W}$ & $1550 \mathrm{~W}$ \\
Plasma gas flow rate & $20.0 \mathrm{~L} \mathrm{~min}^{-1}$ & $15.0 \mathrm{~L} \mathrm{~min}^{-1}$ \\
Carrier gas flow rate & $1.05 \mathrm{~L} \mathrm{~min}^{-1}$ & $1.05 \mathrm{~L} \mathrm{~min}^{-1}$ \\
Plasma sampling & $4 \mathrm{~mm}$ & $10 \mathrm{~mm}$ \\
depth & & \\
Measurement mode & TRA & TRA \\
Dwell time & $20 \mu \mathrm{s}$ & $6 \mathrm{~ms}$ \\
Acquisition time & $60 \mathrm{~s}$ & $100 \mathrm{~s}$ \\
Nebulizer & MicroMist & MicroMist \\
Spray chamber & Cyclonic & Scott double-pass
\end{tabular}

gently for 5 minutes prior to spICP-MS measurement in order to avoid aggregation (NEY Bransonic 300, USA).

Gold nanorods. The gold nanorods were synthesized by the soft template (seed-mediated growth) method described by Zhou et al. ${ }^{20}$ For the sake of stability, finally the CTAB ligand on the surface of the nanorods was exchanged with citrate according to the method described by Mehtala. ${ }^{21}$ This modification was carried out by concentrating the nanorods by ultracentrifugation (13000 rpm for 30 minutes), suspending the nanorods in a $5 \mathrm{~mL}$ portion of a $5 \mathrm{mM}$ trisodium citrate solution and keeping the mixture at room temperature for $12 \mathrm{~h}$. The citrate modification was completed by repeating the process three times. The citrate-coated $\mathrm{Au}$ nanorods were found to be stable for weeks at $25{ }^{\circ} \mathrm{C}$.

The synthesized nanorods were characterized by UV-vis spectroscopy, high resolution TEM and dynamic light scattering (DLS) measurements. The UV-vis plasmonic bands were recorded on a Shimadzu UV-1800 spectrometer, using a quartz cuvette with a one centimeter optical length. The wavelengths of the plasmon peaks in the UV-vis spectra were used to calculate the aspect ratio of the synthesized nanorods. The aspect ratio values obtained in this way agreed well (within 10\%) with the aspect ratios determined by HR-TEM. HR-TEM images were recorded on a Tecnai G2 instrument, using a $200 \mathrm{kV}$ accelerating voltage, and analyzed by using the open-source ImageJ software. In all cases, 10-12 TEM images were taken of all samples, thereby evaluating $c a$. 100 pieces of nanorods, and finally the average length and average width values were calculated along with their standard deviations. The corresponding aspect ratio was calculated using the average data and is given without any standard deviation. DLS measurements were performed at $25 \pm 0.1{ }^{\circ} \mathrm{C}$ with a Zetasizer Nano ZS apparatus (Malvern Instruments, UK) equipped with a He-Ne laser source $(\lambda=633 \mathrm{~nm})$. DLS data were therefore only used as a secondary check on the HR-TEM length data. In order to conserve space here, only representative HR-TEM images of four different aspect ratio gold nanorods are shown in Fig. 1, at two magnifications. DLS measurements only provide hydrodynamic diameters, which correspond reasonably well with the length of nanorods.

Detailed dimensional data for all nanorods in the experiments can be seen in Table 2 . Please note that six different 


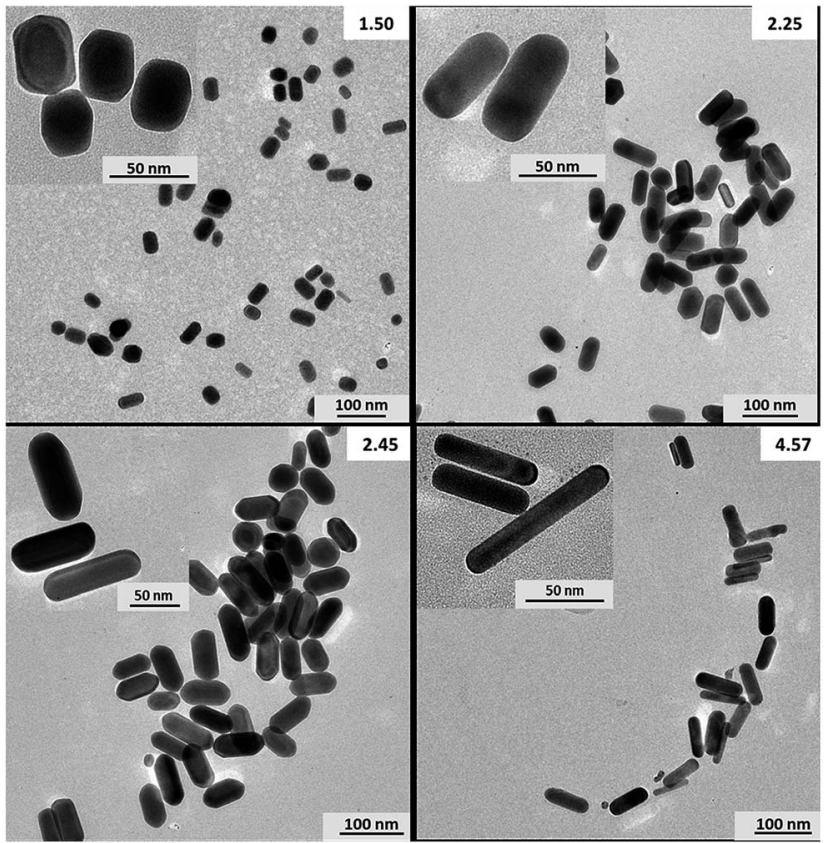

Fig. 1 Representative HR-TEM images of four synthesized gold nanorods, taken using higher and lower magnifications. The respective aspect ratios are indicated in the top right corner of the images.

Table 2 Dimensional data on all gold nanoparticles used in the experiments. Tolerance values represent the standard deviation

Nanospheres

Diameter (nm)

$20.0 \pm 1.8$

$28.8 \pm 3.6$

$39.3 \pm 3.2$

$61.3 \pm 8.7$

$75.4 \pm 9.5$

Nanorods

\begin{tabular}{lll}
\hline Aspect ratio & Width $(\mathrm{nm})$ & Length $(\mathrm{nm})$ \\
\hline 1.50 & $31.2 \pm 3.8$ & $46.9 \pm 9.0$ \\
1.95 & $33.4 \pm 4.6$ & $65.2 \pm 7.6$ \\
2.25 & $29.5 \pm 5.3$ & $66.4 \pm 10.4$ \\
2.45 & $29.0 \pm 6.2$ & $71.2 \pm 11.7$ \\
2.63 & $17.4 \pm 1.2$ & $45.5 \pm 6.3$ \\
3.21 & $28.0 \pm 3.2$ & $89.8 \pm 13.1$ \\
3.80 & $15.5 \pm 1.4$ & $58.4 \pm 4.4$ \\
4.57 & $16.1 \pm 4.6$ & $73.5 \pm 10.7$
\end{tabular}

nanorods were synthesized in our laboratory, while two further nanorods (with aspect ratios of 2.62 and 3.80) were commercially obtained from NanoComposix (USA). In the case of in-lab synthesized nanorods, the data shown in Table 2 were obtained by considering data from all three characterization methods, while data for the two commercial nanorods were taken from their certificates. Please note that due to the special features of the synthesis, some particles with different aspect ratios may have similar widths or lengths.

Gold nanospheres. All spherical gold nanoparticles used in the experiments were commercially obtained from Ted Pella (USA). They were citrate-stabilized PELCO NanoXact particles with nominal diameters of 20, 30, 40, 60 and $75 \mathrm{~nm}$. The exact, certified diameter data of these nanoparticles can be seen in Table 2. The aspect ratio (AR) 1.0 values are indicated for the sake of consistency only.

\subsection{Data evaluation}

spICP-MS measurements on the NexION 350 with 20 microsecond time resolution produced millions of data points during the usual 60-100 s acquisition time of single nanoparticle analysis. Thus pre-processing of the collected data was necessary prior to the statistical evaluation. For this purpose, we developed and used a macro-based program, written in Visual Basic for Applications (Microsoft, USA). This program localized and counted individual particle events in the dataset and calculated their duration. Particle discrimination was based on the condition that a NP peak should be preceded and followed by at least three data points with zero signal. The duration of each NP (ion cloud) transit was then calculated as the product of the number of data points in that event and the dwell time (20 $\mu \mathrm{s})$. The mean transit time was calculated by averaging the individual transit times for only those particles which gave a total particle signal within a $\pm 5 \%$ range of the mode of the signal histogram. The total particle signal for a given NP detection event was obtained as the sum of the time-resolved signals in that event.

The data processing for spICP-MS measurements with normal (millisecond) time resolution and the statistical evaluation of all spICP-MS data was performed using Origin 8.5 (OriginLab, USA) software. Data evaluation in spICP-MS typically involves the plotting of histograms (frequency $v s$. counts diagrams) produced from the collected data points. For Agilent $7700 \times$ TRA data, we use background correction by subtracting the mode of the background peak (fittable by a Poisson function) from the mode of the particle peak (fittable by a lognormal function). The TRA data output by the NexION software has no background (the signal discriminator is set in the software automatically at a relatively high level thereby effectively eliminating the background signal, but also slightly decreasing the sensitivity). All histogram operations were carried out using unit bin sizes. Particle signals in all graphs of the present paper are shown as "characteristic pulse intensity", which refers to the characteristic (total) particle signal at the mode of the histogram peak.

\section{Results and discussion}

\subsection{Effect of particle volume on the NP signal}

It is well established in the spICP-MS literature that the NP signal intensity is proportional to the mass of the particle, which can be converted into its volume by assuming compact particles (bulk density). In the case of spherical particles, which 


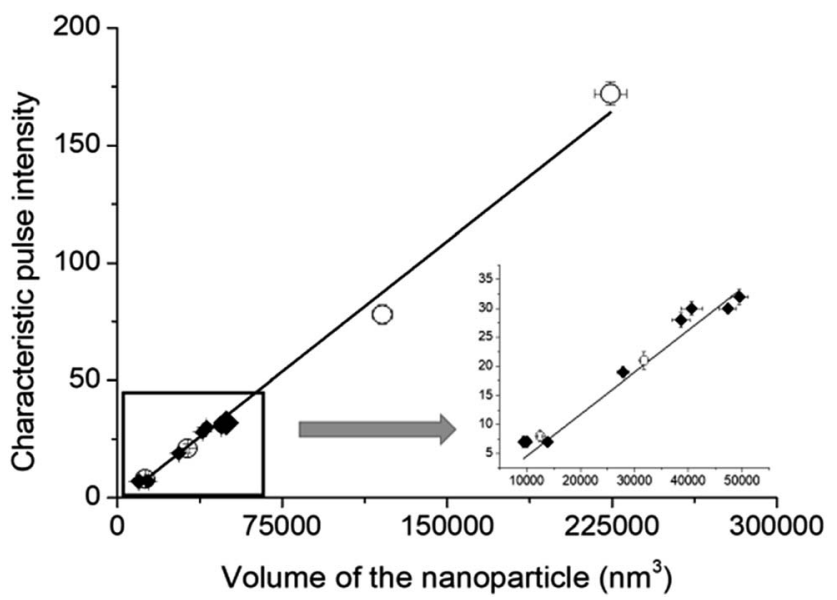

Fig. 2 Joint linear signal vs. particle volume correlation plot for spherical (clear circle) and rod-shaped (black rhombus) nanoparticles, obtained by using normal resolution spICP-MS measurements (dwell time: $6 \mathrm{~ms}$ ). The inset zooms in on the initial part of the plot. Error bars in the $y$ direction indicate standard deviation from three repeated measurements, while in the $x$ direction they are equivalent to 5\% RSD.

is the almost exclusively studied geometry so far, this gives the diameter of the particle..$^{1522-25}$ Based on this, it should also be possible to estimate the volume of nanorod particles by conventional spICP-MS measurements, after size calibration with spherical NP standards.

According to our measurements, the signal from rod-shaped particles indeed fits well to the linear calibration (signal versus volume) plot, as can be seen in Fig. 2. Please note that in all experiments in this study related to particle volume, the average volume of nanorod particles was calculated by considering them to have a cylindrical shape terminated by a hemisphere at each end and using the dimensional data shown in Table 2. Slight inaccuracies of this estimation can explain the small scatter of data points for nanorod particles in the plot. This linear correlation means that the volume of nanorod particles of an unknown size can be assessed by conventional spICP-MS measurements.

It should also be mentioned that the minimum (spherical) particle size that could be detected was estimated to be $24 \mathrm{~nm}$ with the three sigma methodology. This converts to about 7234 $\mathrm{nm}^{3}$ particle volume. At the same time, the smallest volume nanorods used were AR 2.63 and AR 3.80 particles, which had particle volumes of about $10813 \mathrm{~nm}^{3}$ and $11014 \mathrm{~nm}^{3}$, respectively. Thus, the sizes of the nanorods we measured were clearly above the detection limit.

\subsection{Effect of particle geometry on the high resolution signal time profile}

High time resolution makes it possible to assess the transit time of the ion cloud originating from a single $\mathrm{NP}^{\mathbf{2 6 , 2 7}}$ hence the transit time is now known to be in the hundreds of microseconds range. With some of todays ICP-MS instruments, such as the NexION series, 10 or $20 \mu \mathrm{s}$ dwell times are available; thus the transit time can be determined relatively accurately (with e.g. $5 \%$ or less error). The downside is that high time resolution comes with some loss of sensitivity; ${ }^{17}$ for example, even under optimized plasma conditions we could not detect gold nanospheres smaller than $30 \mathrm{~nm}$ on the NexION 350. This loss of sensitivity occurs because the total NP signal is sliced up leaving only a very small signal in each $10-20 \mu$ s dwell time window for small NPs that can be easily buried in background noise. In addition, the signal-to-noise improvement associated with the averaging realized in normal (ms) time resolution can not be effectively used here, as the high resolution signal time profile shows particle-to-particle variation. ${ }^{19}$ These effects are more and more enhanced with decreasing dwell time, which led us to choose $20 \mu \mathrm{s}$ time resolution for our experiments.

Our transit time measurements revealed a fairly linear correlation between the particle diameter and the transit time (Fig. 3) for spherical particles in the studied range. Please note that although it is reasonable to expect that the transit time converges to zero with the decrease of the particle size, here the intercept of the fitted line is slightly positive (the transit time may converge to zero in an order higher than linear). This is probably caused by the combination of several effects, which affects the detection of particles near the size detection limit more severely. First and foremost, the background correction on the NexION slightly overestimates the background level, ${ }^{\mathbf{1 7}}$ which is generally beneficial, but also shortens the measurable transit time of small particles. Second, there are several physical processes in the plasma which influence the transit time of the ion cloud during a detection event: the smaller a particle is, the faster it will be evaporated, meaning that more time is left for the ion cloud to expand during its passage through the plasma via diffusion and mixing. This theoretically increases the transit time, but also decreases the signal intensity in each dwell time window, which may be detrimental for particles near the detection limit.

When examining the transit time of ion clouds from nanorods, the question arises as to whether these particles assume a certain orientation during their flight between the spray

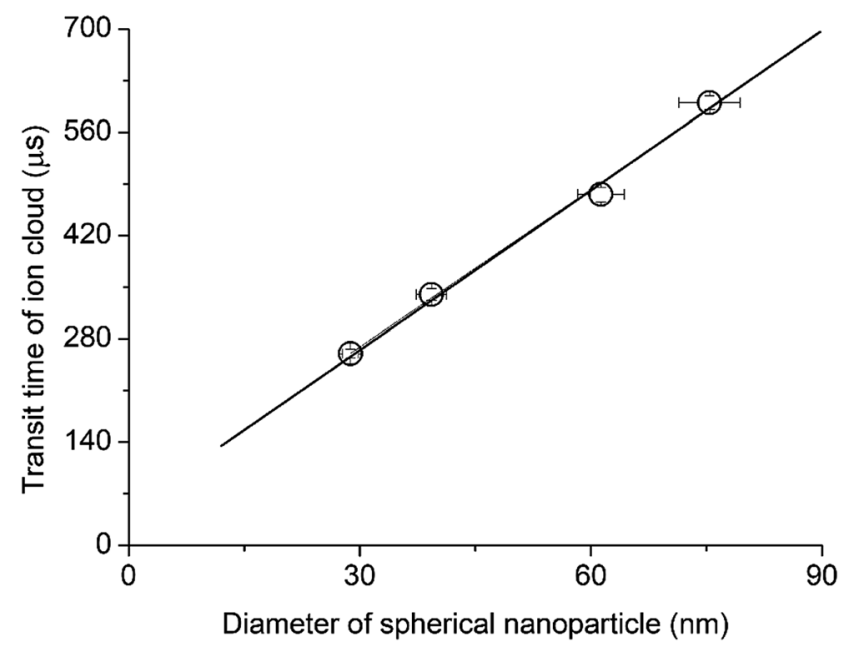

Fig. 3 Linear transit time vs. particle diameter plot for spherical gold NPs, as determined by HR-spICP-MS (dwell time: $20 \mu \mathrm{s}$ ). Error bars in the $y$ direction indicate standard deviation from three repeated measurements, while in the $x$ direction they are equivalent to 5\% RSD. 

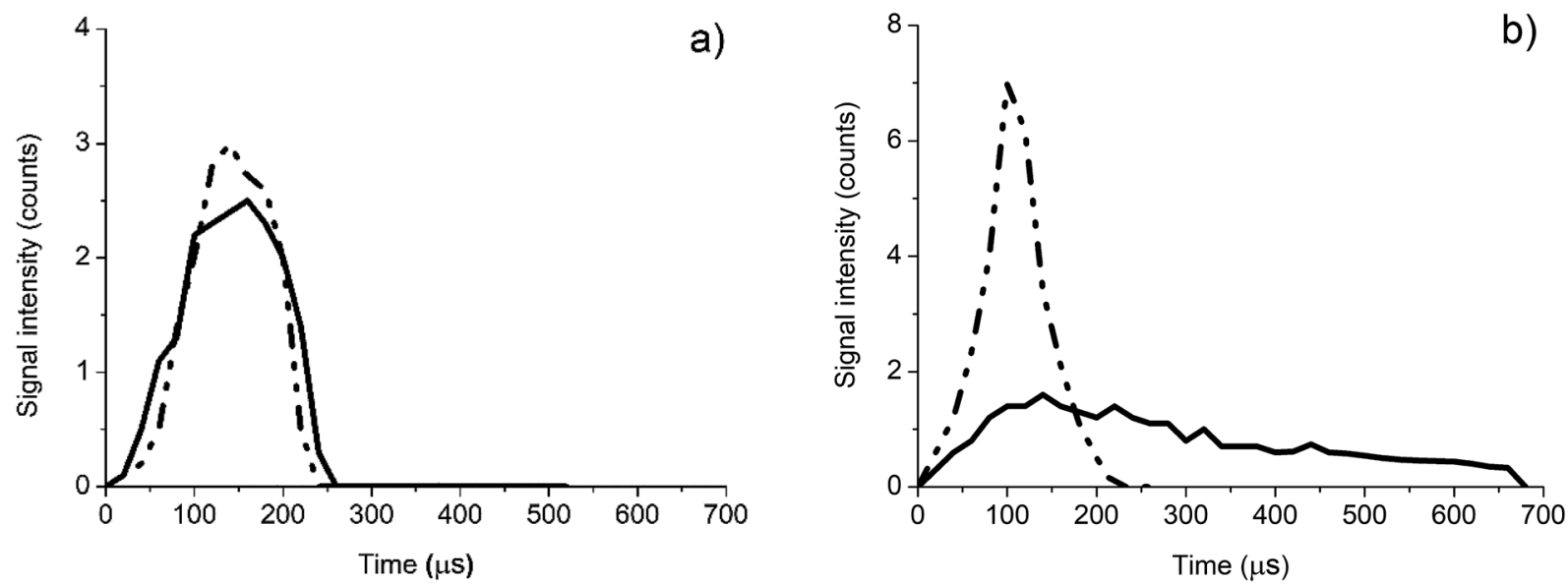

Fig. 4 A comparison of the high time resolution signal time profiles for $30 \mathrm{~nm}$ diameter spherical gold NPs (panel A) and for 2.63 aspect ratio gold nanorods (panel B). In each panel, the two extreme time profiles (with the shortest (dashed dotted line) and longest (solid line) durations observed) as averages for 40-50 particles are shown.

chamber and the plasma or not. This distinct orientation, if it exists, should be the one dictated by aerodynamic effects, namely that the axis of nanorods is aligned with the direction of the carrier gas flow. It has to be considered that there is very little time for such alignment, since it could only occur after the droplet containing the NP in a random orientation is dried (the solvent is evaporated by the radiant heat from the plasma) but before the plasma starts to evaporate the NP material. Thus this notion is highly improbable, but nevertheless it was necessary to experimentally test it due to its relevance. To this end, we statistically evaluated several thousand individual NP time profiles.

For the sake of comparison, the two panels of Fig. 4 show the HR-spICP-MS signal time profiles for spherical and rod-shaped gold NPs. All spherical NPs were found to produce a reasonably Gaussian-shaped signal time profile with very similar durations, whereas time profiles of nanorods were seen to vary randomly in shape between the two extremes represented in Fig. 4b, namely the average profiles for the shortest and the longest transit of the particles. As can be seen in Fig. $4 \mathrm{~b}$, there is a significant

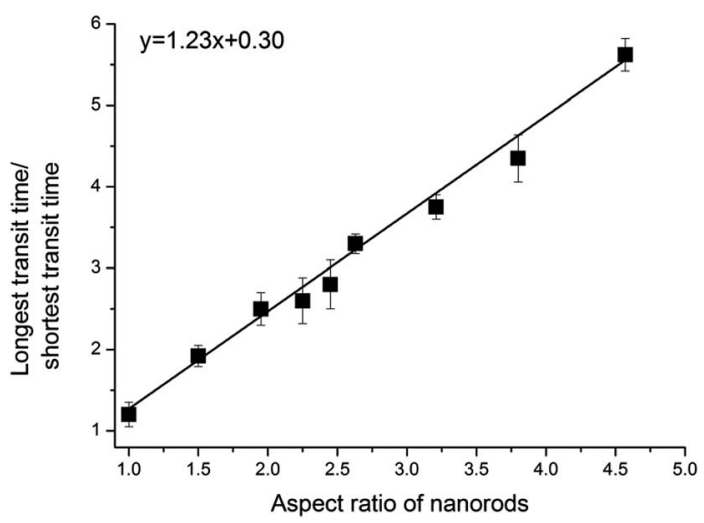

Fig. 5 Correlation between the attributes of the transit time of nanorods and their aspect ratio. Error bars indicate standard deviation from three repeated measurements. difference between the duration of the shortest and the longest transit, although their integrated areas (total NP signal) are similar. This confirms the plausible conjecture that the nanorods are not aligned along the direction of the carrier gas flow during their flight, because if they were then all their individual time profiles should exhibit similar durations. It seems to be reasonable to assume that the shortest duration time profiles belong to nanorods whose longitudinal axis is perpendicular to the direction of propagation ("vertical" in most ICP-MS instruments, where the plasma torch is horizontal), and consequently the longest duration time profiles belong to "horizontally" aligned nanorods. Between these two extremes, all other orientations, and hence signal durations, also occur.

Therefore, the shortest and longest transit times detected for a given nanorod particle are interrelated with the width and the length of the particle, respectively. Although the relationship is probably complex (in addition to the effects discussed above in relation to Fig. 3, now the expansion rate of the ion cloud in the $x, y$ and $z$ directions can also be different due to the shape of the particle), it can be assumed to be describable by a monotonic function. Consequently, the ratio of the longest and shortest transit times can be expected to provide an indication of the aspect ratio (length/width) of the nanorod. As is shown in Fig. 5, our experimental data indeed show a fairly linear correlation between these quantities. Fig. 6 shows the transit time histogram data for the AR 2.25 nanorod sample as an example.

\subsection{Analytical method for the characterization of nanorods}

The correlations described in former sections make it possible to suggest a method for the detection and dimensional analysis of nanorods, based solely on ICP-MS measurements. In the following, we first describe the method and then apply it to the analysis of the hemispherically capped cylindrical nanorods used throughout this study.

The method first requires the determination of the characteristic particle volume $\left(V_{\text {char }}\right)$. This determination needs a few spherical calibration NP standards and normal (ms) resolution 

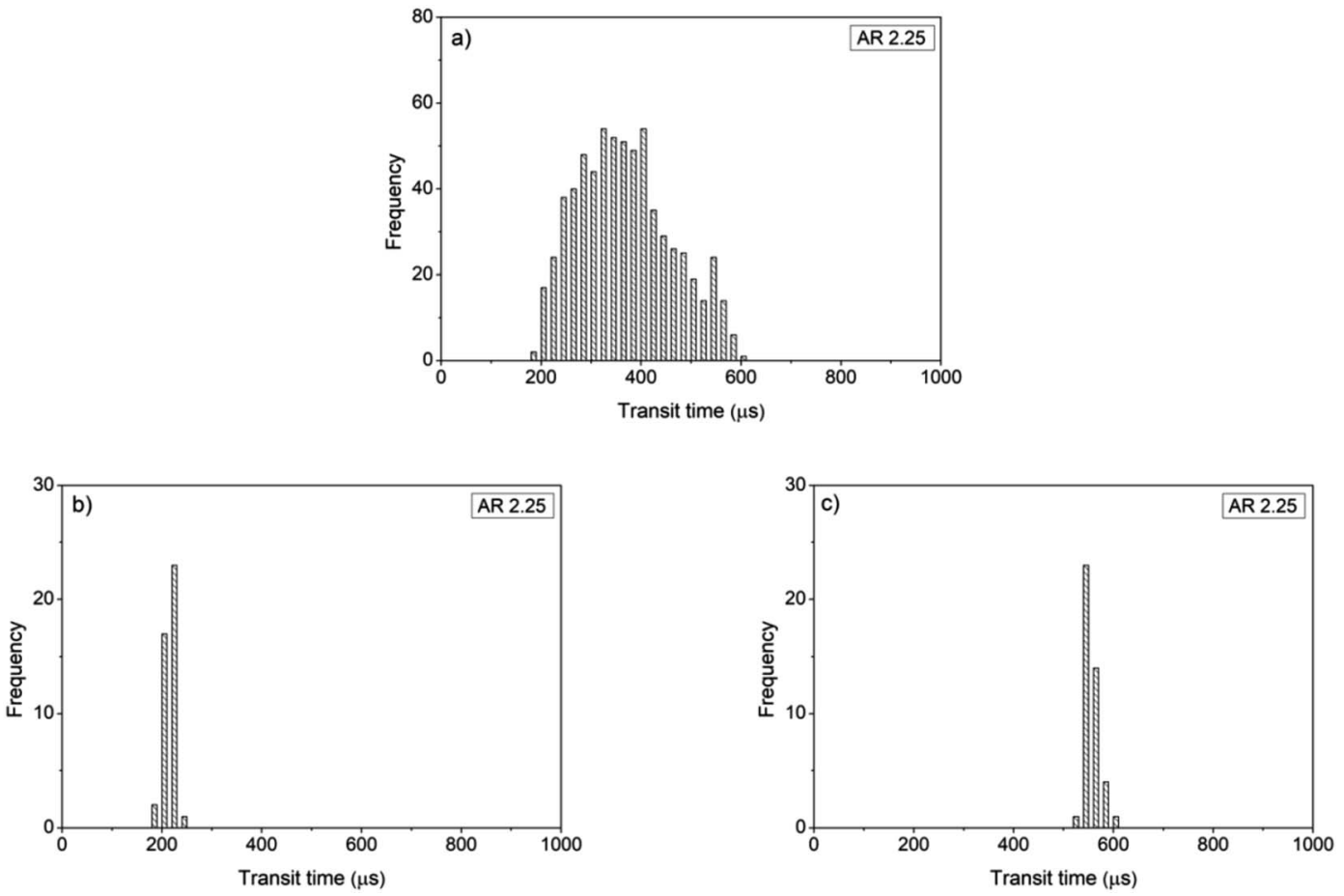

Fig. 6 Transit time histogram data for the AR 2.25 nanorod sample. Panel A: all transit time data, panel B: the shortest 5\% of all transit time data, and panel $\mathrm{C}$ : the longest $5 \%$ of all transit time data.

spICP-MS measurements. As per Fig. 2, the volume determination is based on the characteristic pulse intensity, that is, the signal for the mode of the histogram peak $\left(I_{\text {char }}\right)$. Second, the signal time profiles for the nanorod dispersion need to be recorded using HR-spICP-MS measurements for several thousand particles. The choice between 10 and $20 \mu$ s dwell time should be made with consideration of the NP signal - the $10 \mu \mathrm{s}$ value, which gives a more accurate transit time determination, is preferable provided that the particles are large enough to provide a good S/N signal profile. Through the statistical evaluation of the signal time profiles, the total particle signal and the transit time need to be determined for each detected nanorod particle. The former can be obtained as the sum of the time-resolved signal profile in a detection event. In order to filter the transit time data for the characteristic (most abundant) nanorod particles, only those transit time values have to be retained which gave rise to a particle signal equal to $I_{\text {char }}$ within a reasonable (e.g. $\pm 5 \%)$ tolerance range. This typically leaves at least 700 events in the dataset. Then, the transit time data need to be ordered and the first $N$ and last $N$ values in the list have to be selected and averaged to obtain estimates for the characteristic shortest and longest transit times $\left(t_{\text {short }}\right.$ and $t_{\text {long }}$ respectively). The representativeness can be ensured by assigning a reasonable relative value (e.g. $5 \%$ of the total number of detection events) to $N$, depending on the actual number of detections. The aspect ratio can then be determined based on $t_{\text {long }} / t_{\text {short }}$ and by utilizing the plotted calibration function in Fig. 5. Please note that for maximum accuracy, the establishment of this function may require a fresh calibration to be performed using a few nanorod particle standards, but this is not a must, as the quantities plotted are relative ones and are therefore, to some extent, immune to variations in the sensitivity of the instrument.

It is easy to see that the volume $\left(V_{\text {char }}\right)$ and the aspect ratio (AR) determined above exactly define the characteristic length $(l)$ and width $(w)$ of the nanorod particles. In the case of the hemispherically capped cylindrical nanorods used in the present work, the respective formulas are the following

$$
\begin{gathered}
V_{\text {char }}=\left(\frac{w}{2}\right)^{2} \pi(l-w)+\frac{4}{3}\left(\frac{w}{2}\right)^{3} \pi=\frac{w^{2} l \pi}{2}-\frac{w^{3} \pi}{6} \\
\mathrm{AR}=\frac{l}{w}
\end{gathered}
$$

the solving of which for $w$ and $l$ gives

$$
\begin{gathered}
w=\sqrt[3]{\frac{6 V_{\text {char }}}{(3 \mathrm{AR}-1) \pi}} \\
l=\mathrm{AR} \sqrt[3]{\frac{6 V_{\text {char }}}{(3 \mathrm{AR}-1) \pi}}
\end{gathered}
$$

Of course, the geometry calculations described here can be adapted for other nanorod shapes too (e.g. purely cylindrical nanorods or nanorods with a star-shaped cross section, etc.). Note that the above formulas are also applicable to the case of spherical particles $(\mathrm{AR}=1.0)$. 
Table 3 Calculated width and length data for nanorods as determined by high resolution spICP-MS measurements. The relative error is calculated with respect to the data shown in Table 2

\begin{tabular}{llll}
\hline $\begin{array}{l}\text { Width } \\
(\mathrm{nm})\end{array}$ & $\begin{array}{l}\text { Length } \\
(\mathrm{nm})\end{array}$ & $\begin{array}{l}\text { Rel. error for } \\
\text { the width }(\%)\end{array}$ & $\begin{array}{l}\text { Rel. error for } \\
\text { the length }(\%)\end{array}$ \\
\hline 30.9 & & & 1.0 \\
32.9 & 47.5 & 1.4 & 1.6 \\
30.3 & 66.7 & 2.3 & 2.7 \\
29.9 & 63.7 & 4.3 & 3.0 \\
17.2 & 67.8 & 4.9 & 1.1 \\
28.9 & 46.3 & 1.7 & 3.1 \\
15.9 & 85.2 & 5.4 & 2.7 \\
15.9 & 55.7 & 4.8 & 1.9 \\
\hline
\end{tabular}

For our nanorod samples, Table 3 lists the particle length and width data determined by the above ICP-MS method, as well as the error of determination with reference to the actual dimensions of the nanorods (Table 2).

As can be seen, the accuracy of the method is quite reasonable (the relative inaccuracy is below 6\%), which compares favourably with the relative inaccuracy of the reference values. The measurement time is also short; once the calibration of the instrument is done, each sample can be analyzed in 3-5 minutes. The only sample preparation is dilution, if it is needed at all. Please note that the added benefit of a purely ICP-MS based analysis is that it can also provide information about (i) the number concentration of the dispersion and (ii) the composition of the NPs.

It can also be added that the accuracy of the method can be enhanced by increasing the number of detected and processed particle signals. Although it is usually already in the range of thousands in a couple of minutes of acquisition time, the use of high time resolution can further boost this number. The reason for this is that the minimum time separation between incoming particles needed to observe separate NP detection events is reduced; instead of a few milliseconds, it can be as little as a few times $10 \mu \mathrm{s}$. This may allow the analyst to work with dispersions that have as much as two orders of magnitude higher number concentration (e.g. $10^{6}$ to $10^{7} \mathrm{~mL}^{-1}$ instead of $10^{5} \mathrm{~mL}^{-1}$ ), thereby largely increasing the number of detected particles during the same acquisition time.

\section{Conclusions}

For the first time in the literature, a systematic investigation of the signal time profiles recorded with high temporal resolution (20 $\mathrm{\mu s}$ ) for rod-shaped nanoparticles was performed. The experiments were carried out on hemispherically capped cylindrical gold nanorods synthesized in the aspect ratio range from 1.5 to 4.5. A comparison of NP signals and time profiles for spherical and rod-shaped NPs revealed that (i) the volume of the particles can be assessed by conventional spICP-MS measurements using a joint, linear calibration plot; and (ii) the shape of time profiles and transit times for equal-volume spherical and rod-shaped NPs are different, based on which the aspect ratio can be calculated.
By utilizing the above relationships, a new analytical method, based solely on ICP-MS measurements, was developed for the detection and dimensional analysis of nanorod-shaped NPs. The method combines data from normal (ms-range) and high ( $\mu$ s-range) time resolution spICP-MS measurements. The accuracy of the method was assessed by employing it for the dimensional analysis of eight gold nanorods with different aspect ratios. It was shown that the length and width of the particles can be determined with $6 \%$ error or less. Please note that since the similarity of nanoparticles in any batch is always limited, the reasonable expectation can only be to obtain characteristic values for the dimensions, describing the most abundant particles (mode of the size distribution histogram). Although the method was here only tested for hemispherically capped cylindrical gold nanorods, it should be easy to apply the method to other nanorods too.

\section{Conflicts of interest}

There are no conflicts to declare.

\section{Acknowledgements}

I. Kálomista, A. Kéri and G. Galbács gratefully acknowledge the EU-funded Hungarian grant (No. EFOP-3.6.2-16-2017-00005) that supported this research. The research was also supported by the National Research, Development and Innovation Office (NKFIH) through projects No. K116323 and No. GINOP-2.3.2-152016-00013 and GINOP-2.3.2-15-2016-00038, as well as by the János Bolyai Research Scholarship of the Hungarian Academy of Sciences (E. Csapó).

\section{References}

1 A. S. Aricò, P. Bruce, B. Scrosati, J.-M. Tarascon and W. van Schalkwijk, Nat. Mater., 2005, 4, 366-377.

2 S. D. Caruthers, S. A. Wickline and G. M. Lanza, Curr. Opin. Biotechnol., 2007, 18, 26-30.

3 A. T. Bell, Science, 2003, 299, 1688-1691.

4 P. D. Howes, R. Chandrawati and M. M. Stevens, Science, 2014, 346, 1247390.

5 N. R. Jana, L. Gearheart and C. J. Murphy, Adv. Mater., 2001, 13, 1389-1393.

6 L. Vigderman, B. P. Khanal and E. R. Zubarev, Adv. Mater., 2012, 24, 4811-4841.

7 M. A. Mahmoud, D. O'Neil and M. A. El-Sayed, Nano Lett., 2014, 14, 743-748.

8 X. Huang, S. Neretina and M. A. El-Sayed, Adv. Mater., 2009, 21, 4880-4910.

9 J. Stone, S. Jackson and D. Wright, Nanomedicine, 2011, 3, 100-109.

10 J. Perez-Juste, I. Pastoriza-Santos, L. M. Liz-Marzan and P. Mulvaney, Coord. Chem. Rev., 2005, 249, 1870-1901.

11 J. Perez-Juste, L. M. Liz-Marzan, S. Carnie, D. Y. C. Chan and P. Mulvaney, Adv. Funct. Mater., 2004, 14, 571-579. 
12 C. J. Murphy, T. K. Sau, A. M. Gole, C. J. Orendorff, J. Gao, L. Gou, S. E. Hunyadi and T. Li, J. Phys. Chem. B, 2005, 29, 13857-13870.

13 M. D. Montaño, J. W. Olesik, A. G. Barber, K. Challis and J. F. Ranville, Anal. Bioanal. Chem., 2016, 408, 5053-5074.

14 S. Lee, X. Bi, R. B. Reed, J. F. Ranville, P. Herckes and P. Westerhoff, Environ. Sci. Technol., 2014, 48, 10291-10300. 15 I. Kálomista, A. Kéri and G. Galbács, J. Anal. At. Spectrom., 2016, 31, 1112-1122.

16 I. Kálomista, A. Kéri and G. Galbács, Talanta, 2017, 172, 147154.

17 M. D. Montaño, H. R. Badiei, S. Bazargan and J. F. Ranville, Environ. Sci.: Nano, 2014, 1, 338-346.

18 A. Hineman and C. Stephan, J. Anal. At. Spectrom., 2014, 29, 1252-1257.

19 J. Liu, K. E. Murphy, R. I. MacCuspie and M. R. Winchester, Anal. Chem., 2014, 86, 3405-3414.
20 N. Zhou, L. Polavarapu, N. Gao, Y. Pan, P. Yuan, Q. Wangbc and Q.-H. Xu, Nanoscale, 2013, 5, 4236-4241.

21 J. G. Mehtala, D. Y. Zemlyanov, J. P. Max, N. Kadasala, S. Zhao and A. Wei, Langmuir, 2014, 30, 13727-13730.

22 C. Degueldre and P.-Y. Favarger, Colloids Surf., A, 2003, 217, 137-142.

23 F. Laborda, M. S. Jiménez, E. Bolea and J. Castillo, Int. J. Mass Spectrom., 2011, 307, 99-104.

24 F. Laborda, E. Bolea and J. Jiménez-Lamana, Anal. Chem., 2014, 86, 2270-2278.

25 H. E. Pace, N. J. Rogers, C. Jarolimek, V. A. Coleman, E. P. Gray, C. P. Higgins and J. F. Ranville, Environ. Sci. Technol., 2012, 46, 12272-12280.

26 S. Gschwind, L. Flamigni, J. Koch, O. Borovinskaya, S. Groh, K. Niemax and D. Gunther, J. Anal. At. Spectrom., 2011, 26, 1166-1174.

27 J. Koch, L. Flamigni, S. Gschwind, S. Allner, H. Longerichb and D. Gunthera, J. Anal. At. Spectrom., 2013, 28, 1707-1717. 